\title{
Target energy effects on Type 1 and Type 2 visual persistence
}

\author{
GERAL M. LONG and PAUL R. McCARTHY \\ Villanova University, Villanova, Pennsylvania 19085
}

\begin{abstract}
The effects of varying target energy (luminance and duration) were determined for two different types of visual persistence. Type 1 persistence, which reflects the latency for perceived offset of a brief stimulus, was found to be inversely related to target energy. Type 2 persistence, which reflects the total perceived duration of the fading stimulus trace, was found to be positively related to target energy. These results for both types of persistence were established with the common asynchrony-judgment task and with a simple reaction time task. The opposite relationships for the two types of visual persistence with target-energy manipulations were discussed in terms of: (1) the different processes believed to underlie Type 1 and Type 2 persistences and (2) the disparate empirical results frequently reported in the current persistence literature.
\end{abstract}

In the current visual persistence literature, there is extensive theoretical and empirical confusion. A clear indication of this condition can be seen in two recent reviews that have reached very different conclusions concerning the nature and locus of persistence effects within the visual system (Coltheart, 1980; Long, 1980). A significant portion of this confusion may be attributed to the empirical discrepancies that seem to plague this literature. For example, it has been reported that persistence decreases with increasing target luminance (e.g., Bowen, Pola, \& Matin, .1974; Efron, 1970; Hansteen, 1971; Long \& Gildea, 1981), is independent of changes in target luminance (e.g., Adelson \& Jonides, 1980; Hogben \& DiLollo, 1974), and increases with increasing target luminance (e.g., Keele \& Chase, 1967; Long \& Sakitt, 1980, 1981). Similar contradictory claims can be found concerning the effects of varying target duration and background luminance (cf. Long, 1980).

One recent, and potentially fruitful, attempt to reconcile such discrepancies has suggested that there may exist more than a single type of visual persistence (e.g., Hawkins \& Shulman, 1979; Sakitt \& Long, 1979). In this conceptualization, following the physical offset of a brief target, two distinguishable phenomenal events may be available to an observer. The first is the perceived offset of the stimulus, which follows the physical offset of the stimulus very rapidly; the second is the subjectively fading trace of the stimulus, which may persist for several hundred milliseconds. Following the proposal by Hawkins and Shulman (1979), the former will be termed Type 1 persistence and the latter, Type 2 persistence. The value of such a dichotomy rests on the critical proposal that Type 1 persistence exhibits inverse energy

The data presented here were collected as part of the second author's master's thesis at Villanova University. This research was supported in part by NSF Grant TFI-8100524 to the first author. effects, whereas Type 2 persistence exhibits positive energy effects (e.g., Hawkins \& Shulman, 1979). It has further been suggested (Long, 1979) that the two persistences may be related to identifiable physiological processes: Type 1 persistence to the latency for "off" responses (which is inversely related to target energy) and Type 2 persistence to prolonged photoreceptor activity (which is positively related to target energy). Hence, in this dual-model view of persistence, the apparent discrepancies in the persistence literature may be attributed to the inadvertent assessment of different types of persistence across experimental studies.

The purpose of the present note is twofold. First, it seeks to provide further clear evidence for the proposed dichotomized treatment of visual persistence. The effects of varying luminance and duration on the two types of persistence will be assessed under "typical" viewing conditions. Second, the comparability of two standard persistence tasks will be determined. On both the temporal asynchrony task (e.g., Efron, 1970) and the simple RT task of persistence (e.g., Parker, 1980), demonstrations of Type 1 and Type 2 persistence will be attempted. The rationale for this comparison can be found in the recent suggestion by Coltheart (1980) that the RT and asynchrony tasks of persistence may not be equivalent measures of persistence because of different levels of stimulus processing to which each may be sensitive.

\section{METHOD}

The target stimulus in all cases was a 1.0-deg black dot centrally located in the $7.6 \times 4.5 \mathrm{deg}$ white field of a Scientific Prototype three-channel tachistoscope (Model 320-GB). This target was presented at three duration levels $(25,50$, and $100 \mathrm{msec}$ ) and two luminance levels (5.0 and $15 \mathrm{fL})$. The white background field that preceded and followed the target presentation was constant at $5 \mathrm{fL}$. For the asynchrony-judgment task, three Hunter Timers (Model III-C) were interfaced with the tachistoscope to provide a constant $500-\mathrm{msec}$ foreperiod 
between warning signal and target presentation, a variableduration interval (adjusted by the experimenter) between the offset of the target and the onset of the auditory probe, and a constant 50 -msec auditory probe. The probe was a $1,000-\mathrm{Hz}$ tone presented by a Lafayette audio generator and amplifier (Model 15010). For the RT task, a Hunter Klockounter (Model 120A) was initiated at the offset of the target stimulus and was stopped when the observer released a telegraph key.

Five graduate students who were naive with respect to the experimental hypotheses served as paid observers. Each observer was run in four experimental sessions, each of which was preceded by a 60-min practice session with the identical conditions employed in the experimental session to follow. The four experimental sessions consisted of four different task conditions: (1) RT to offset, (2) asynchrony to offset, (3) RT to persistence, and (4) asynchrony to persistence. The first two conditions represent theoretical Type 1 persistence because the observer was instructed to attend to the perceived offset of the target. With the RT task, the observer was instructed to release the telegraph key on each trial as soon as the brief target was terminated. With the asynchrony task, the experimenter adjusted the auditory probe in ascending or descending $50 \mathrm{msec}$ steps until the observer reported that it coincided with the offset of the brief target. (The latter procedure has been used previously by Long and Gildea, 1981.)

In the other two experimental conditions, theoretical Type 2 persistence was assessed. For both, observers were instructed to attend to any lingering trace of the brief target. With the RT task, the observer released the telegraph key when all trace of the target had completely disappeared. With the asynchrony task, the experimenter adjusted the auditory probe in ascending or descending $50-\mathrm{msec}$ steps until the observer reported that it coincided with the end of any persistence from the brief target. (The latter procedure has been used previously by Long and Beaton, 1980a, 1980b, and Long and Sakitt, 1981.)

Within each of the four task conditions, six conditions of the target stimulus were employed (three durations $X$ two luminances). For the two sessions employing RT procedures, the six target conditions were represented by six randomly ordered blocks of 12 trials. For the two sessions employing asynchrony procedures, the six target conditions were represented by six randomly ordered blocks of 10 trials. Half of these asynchronyjudgment trials involved ascending $50-\mathrm{msec}$ steps; half involved descending 50 -msec steps. The order of the four experimental sessions (i.e., task conditions) was uniquely randomized for each observer. Each experimental session lasted 60-90 min, with a brief rest period halfway through the session.

\section{RESULTS AND DISCUSSION}

The mean results for the five observers in the four task conditions are shown in the four panels of Figure 1. The top two panels presumably represent Type 1 persistence as assessed by the RT task (Figure 1a) and the asynchrony task (Figure 1b). For both these tasks, the observers were explicitly instructed to attend the target offset. Note that for both tasks, the effects of increasing target luminance or target duration were to decrease the response latency. For the RT task, significant main effects of luminance $[F(1,4)=35.26, p<.005]$ and duration $[F(2,8)=249.32, p<.001]$ were obtained; the interaction was not significant $[F(2,8)=4.10$, $p>.05]$. For the asynchrony task, the main effects of luminance $[F(1,4)=31.55, p<.005]$ and duration $[F(2,8)=35.67, p<.001]$ were again significant; no interaction was indicated $[\mathrm{F}(2,8)=1.49, \mathrm{p}>.05]$. Hence, an inverse energy effect was obtained on both

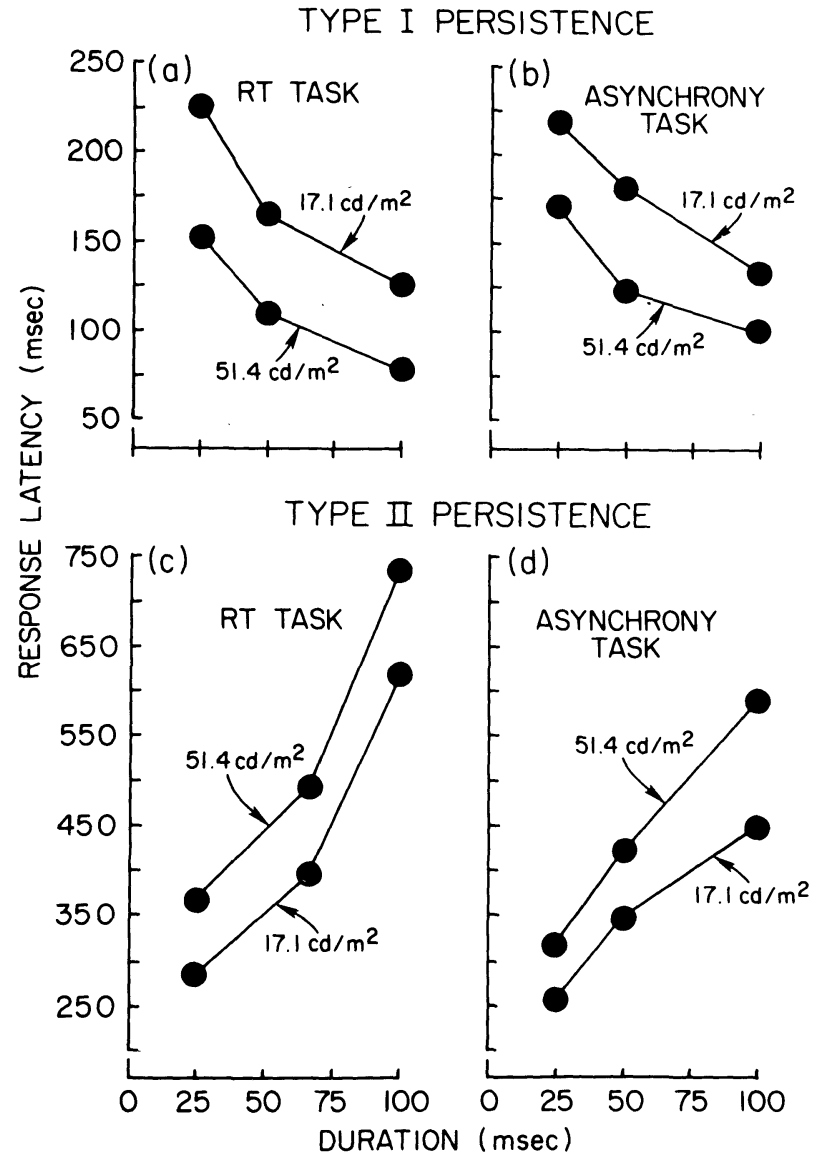

Figure 1. Mean response latencies on the four persistence tasks as a function of target duration for two luminance levels. Figures $1 \mathrm{a}$ and $1 \mathrm{~b}$ correspond to Type 1 persistence, in which observers attended to target offset. Figures $1 \mathrm{c}$ and $1 \mathrm{~d}$ correspond to Type 2 persistence, in which observers attended to fading target image.

tasks under the same instructional sets. This entire pattern of results is consistent with predictions for Type 1 persistence and with many previous investigations with either of these procedures that have reported inverse luminance and duration effects on persistence (cf. Bowen, 1981; Coltheart, 1980).

The latency values in Figure 1a (RT to offset) may appear suspiciously low for motor response times. However, a control condition with two observers has shown that, if an observer is instructed to respond to target onset, the latency values (i.e., RT-target duration) are $50-125$ msec less than those shown in the figure. Nonetheless, observers did report considerable difficulty on the RT task responding to offset as distinct from onset for the briefest $(25-\mathrm{msec})$ targets. This may represent a problem of a "cellar effect" for the RT task under some conditions that was apparently absent on the asynchrony task. However, when target duration was increased to longer values, this reported difficulty largely disappeared, and the same inverse energy effects were obtained (e.g., Briggs \& Kinsbourne, 1972; Parker, 1980). 
The bottom two panels in Figure 1 theoretically represent Type 2 persistence as assessed by the RT task (Figure 1c) and asynchrony task (Figure 1d). For both these tasks, the observers were explicitly instructed to attend to any fading trace of the target stimulus after its offset. Note, first, the much higher latency values in the bottom panels than in the upper panels for the same response tasks and stimulus conditions. This reflects the much greater persistence for Type 2 persistence, which can outlast perceived offset by hundreds of milliseconds. Second, in contrast to the upper panels in the figure, a positive effect of increasing either target luminance or target duration was obtained for both tasks. For the RT task, the main effects for luminance $[F(1,4)=33.49, p<.005]$ and duration $[F(2,8)=6.98$, $\mathrm{p}<.025$ ] were significant; the interaction was not significant $(F<1.0)$. Similarly, for the asynchrony task, the main effects for luminance $[\mathrm{F}(1,4)=37.55$, $\mathrm{p}<.005]$ and duration $[\mathrm{F}(2,8)=11.95, \mathrm{p}<.005]$ were again significant; the interaction was not significant $[F(2,8)=3.99, p>.05]$. This pattern of results is consistent with predictions for Type 2 persistence outlined above and with several previous investigations that have obtained positive energy effects upon visual persistence (cf. Long, 1980).

An additional concern of the present study was the comparability of the RT and asynchrony tasks for assessing visual persistence. It would appear from Figure 1 that at least under the present conditions, the two procedures are basically equivalent, regardless of whether Type 1 or Type 2 persistence is assessed. This is not to say that the absolute values obtained with the two tasks are identical. After all, different responses are required by the two tasks. However, the relative effect of manipulating stimulus variables appears to be equivalent across the RT and asynchrony tasks of visual persistence.

\section{CONCLUSION}

From the results obtained in the present work, it would appear that the distinction between Type 1 and Type 2 persistence is not only readily demonstrable but also of clear empirical importance. Under normal photopic viewing conditions, observers have little difficulty distinguishing between the two phenomenal events of perceived offset and the end of persistence that follow a brief target stimulus. Moreover, the very different empirical relationships exhibited by the two persistences in response to manipulation of target luminance and target duration indicate that very different processes in the visual system underlie the phenomenally distinct events. And finally, the recognition of these separate types of persistence permits a reconciliation of the current persistence literature that is otherwise replete with numerous empirical contradictions.

\section{REFERENCES}

Adelson, E. H., \& Jonides, J. The psychophysics of iconic storage. Journal of Experimental Psychology: Human Perception and Performance, 1980, 6, 486-493.

Bowen, R. W. Latencies for chromatic and achromatic visual mechanisms. Vision Research, 1981, 21, 1457-1466.

Bowen, R. W., Pola, J., \& Matin, L. Visual persistence: Effects of flash luminance, duration and energy. Vision Research, 1974, 14, 295-303.

Briggs, G., \& Kinsbourne, M. Visual persistence as measured by reaction time. Quarterly Journal of Experimental Psychology, 1972, 24, 318-325.

Coltheart, M. Iconic memory and visible persistence. Perception \& Psychophysics, 1980, 27, 183-228.

EFron, R. The relationship between the duration of a stimulus and the duration of a perception. Neuropsychologia, 1970, 8, 37-55.

HANSTEE N, R. W. Visual latency as a function of stimulus onset, offset, and background luminance. Journal of the Optical Society of America, 1971, 61, 1190-1195.

Hawkins, H. L., \& Shulman, G. L. Two definitions of persistence in visual perception. Perception \& Psychophysics, 1979, 25, 348-350.

Hogben, J. H., \& Dilollo, V. Perceptual integration and perceptual segregation of brief visual stimuli. Vision Research, $1974,14,1059-1069$.

Keele, S. W., \& Chase, W. G. Short-term visual storage. Perception \& Psychophysics, 1967, 2, 383-386.

Long, G. M. Comment on Hawkins and Shulman's Type I and Type II visual persistence. Perception \& Psychophysics, 1979, 26, 412-414.

LoNG, G. M. Iconic memory: A review and critique of the study of short-term visual storage. Psychological Bulletin, 1980, 88, 785-820.

Long, G. M., \& Beaton, R. J. The contribution of visual persistence to the perceived duration of brief targets. Perception \& Psychophysics, 1980, 28, 422-430. (a)

Long, G. M., \& BEATon, R. J. The effects of spatial frequency and target type on perceived duration. Perception \& Psychophysics, 1980, 28, 413-421. (b)

Long, G. M., \& GildeA, T. J. Latency for the perceived offset of brief target gratings. Vision Research, 1981, 21, 1395-1399.

Long, G. M., \& SAKiTt, B. The retinal basis of iconic memory: Eriksen and Collins revisited. American Journal of Psychology, 1980, 93, 195-206.

LoNG, G. M., \& SAKitT, B. Differences between flicker and nonflicker persistence tasks: The effects of luminance and the number of cycles in a grating target. Vision Research, 1981, 21, 1387-1393.

Parker, D. M. Simple reaction times to the onset, offset, and contrast reversal of sinusoidal grating stimuli. Perception \& Psychophysics, 1980, 28, 365-368.

Sakitт, B., \& Long, G. M. Cones determine subjective offset of a stimulus but rods determine total persistence. Vision Research, 1979, 19, 1439-1441.

(Received for publication January 26, 1982.) 